\title{
FRD characterization in large-scale for FOCCoS of Prime Focus Spectrograph for Subaru telescope
}

Antonio Cesar de Oliveira, James E. Gunn, Yuki Moritani, Ligia Souza de Oliveira, Leandro Henrique dos Santos, et al.

Antonio Cesar de Oliveira, James E. Gunn, Yuki Moritani, Ligia Souza de Oliveira, Leandro Henrique dos Santos, Daniel Reiley, Brent Belland, Naoyuki Tamura, Naruhisa Takato, Laerte Sodré Junior, Bruno Castilho, David L. Keith, "FRD characterization in large-scale for FOCCoS of Prime Focus Spectrograph for Subaru telescope ," Proc. SPIE 10702, Ground-based and Airborne Instrumentation for Astronomy VII, 107027R (9 July 2018); doi: 10.1117/12.2312287

Event: SPIE Astronomical Telescopes + Instrumentation, 2018, Austin, Texas, United States 


\title{
FRD characterization in large-scale for FOCCoS of Prime Focus Spectrograph for Subaru telescope
}

\author{
Antonio Cesar de Oliveira ${ }^{1}$, James E. Gunn ${ }^{2}$, Yuki Moritani ${ }^{3}$, Ligia Souza de Oliveira ${ }^{4}$, Leandro \\ Henrique dos Santos ${ }^{4}$, Daniel Reiley ${ }^{5}$, Brent Belland ${ }^{5}$, Naoyuki Tamura ${ }^{3}$, Naruhisa Takato ${ }^{6}$, Laerte \\ Sodré Junior ${ }^{7}$, Bruno Castilho ${ }^{1}$, David L. Keith ${ }^{8}$.
}
1- MCT/LNA -Laboratório Nacional de Astrofísica, Itajubá - MG - Brasil
2- Princeton University - Princeton - Brasil

3- Kavli Institute for the Physics and Mathematics of the Universe (WPI), University of Tokyo Institutes for Advanced Study, The University of Tokyo, Kashiwa, Chiba 277-8583, Japan IPMU University of Tokyo, Institute for the Physics and Mathematics of the Universe Tokyo - Japan

4- OIO - Oliveira Instrumentação Óptica - MG and SP - Brasil

5- Caltech - USA

6- Subaru Telescope/ National Astronomical Observatory of Japan

7- IAG/USP - Instituto Astronômico e Geográfico/ Universidade de São Paulo - SP - Brasil

8- Independent Consultant -WA - USA

\begin{abstract}
The focal ratio degradation effects on optical fibers, technically referred to as FRD, has been the subject of intense studies since the beginning of the use of optical fibers in the construction of instruments applied in astronomy. A number of studies attempt to relate FRD to light loss in the optical system and other studies attempt to qualify and quantify FRD as a function of the stress induced during assembly of the structures supporting the ends of the optical fibers. In this work, we present a large-scale study to characterize FRD in all the fibers that make up the cables of the FOCCoS, Fiber Optical Cable and Connectors System project. FOCCoS, has the main function of capturing the direct light from the focal plane of Subaru Telescope using 2400 optical fibers, each one with a microlens in its tip, and conducting this light through a route containing connectors to a set of four spectrographs. The optical fiber cable is divided in 3 different segments called Cable A, Cable B and Cable C. Multi-fibers connectors assure precise connection among all optical fibers of the segments, providing flexibility for instrument changes. Our study provides procedures and methods to analyze the effects of FRD on all cable segments for each type of termination involved. Special attention is devoted to the understanding of how angular deviations between the input surface of the fiber and the test beam can significantly influence the calculation of FRD in optical fibers.
\end{abstract}

\section{INTRODUCTION}

The Focal Ratio Degradation effect on optical fibers is naturally a consequence of imperfections of the manufacturer. However, any type of pressure or curvature beyond a critical level causes stress and as a consequence increase the natural FRD of the fiber. Another important factor to consider are the building procedures. Details such as, type of epoxy, the amount of epoxy and the polishing process are of extreme importance within a suitable conduit to avoid the increment of FRD in the fibers. In general, fiber termination can present two types of problems; The first due to the stress of the assembly and the second due to the polishing. The stress of the assembly generally occurs by virtue of

Ground-based and Airborne Instrumentation for Astronomy VII, edited by Christopher J. Evans, Luc Simard, Hideki Takami, Proc. of SPIE Vol. 10702, 107027R · C 2018 SPIE · CCC code: 0277-786X/18/\$18 · doi: 10.1117/12.2312287 
mechanical pressure on the fiber by the ferrule or the groove channels which support the termination. It can also be caused by excess of epoxy or by the kind of epoxy. An epoxy with a big constriction effect obviously cements the fiber over pressure maintaining constant stress. On the other hand, the polishing of fiber terminations can introduce angular errors. This happens most of the time because of imprecision in the polishing jigs, but it can also happen due to some imprecision of structural fitting. In this context, the assembly of fiber optic ends inserted in cylindrical ferrules with internal diameter matched to the fiber diameter does not offer great problems of angular deviation. In addition, the industrial polishing jigs are quite precise in their mechanism of keeping the ferrules fixed during the polishing. FOCCoS contains three types of terminations for the fibers used in its construction. The first termination is a linear arrangement which constitutes the Slit device $^{(01)}$. The second type of termination is defined by zirconia ferrules, Fig.1, which are used at the light collecting ends together with individuals microlenses ${ }^{(02)}$. The third is defined by $32 \mathrm{MTP}$ ferrules produced by USCONEC company ${ }^{(03,04)}$. This latter is a multi-fiber connection cell that can house up to 32 ends of optical fibers, Fig. 2. In this work, we drive the experiments using optical fiber cables ended with this type of connection cell. The analysis of FRD taking special care with angular deviation in these samples proved to be extremely useful to characterize this kind of arrangement.

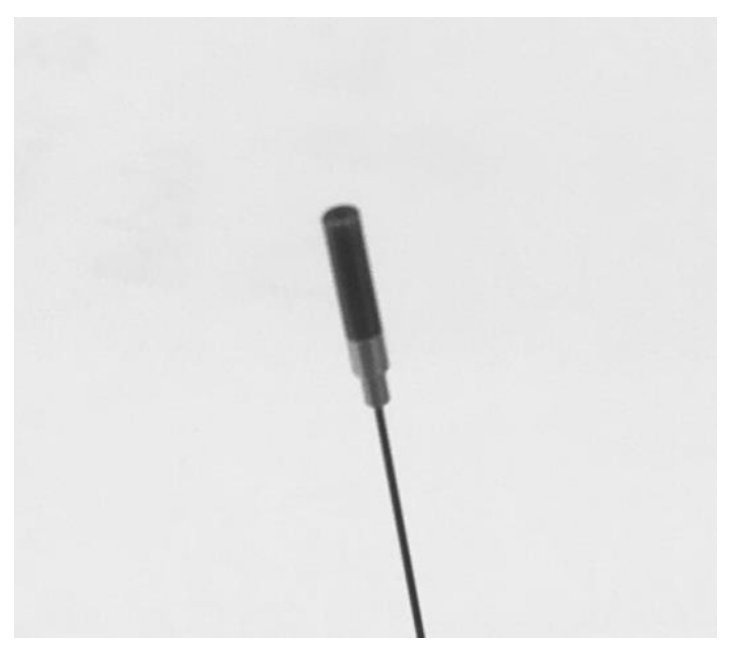

Figure 1- Photograph of a Zirconia ferrule with a fiber inserted.

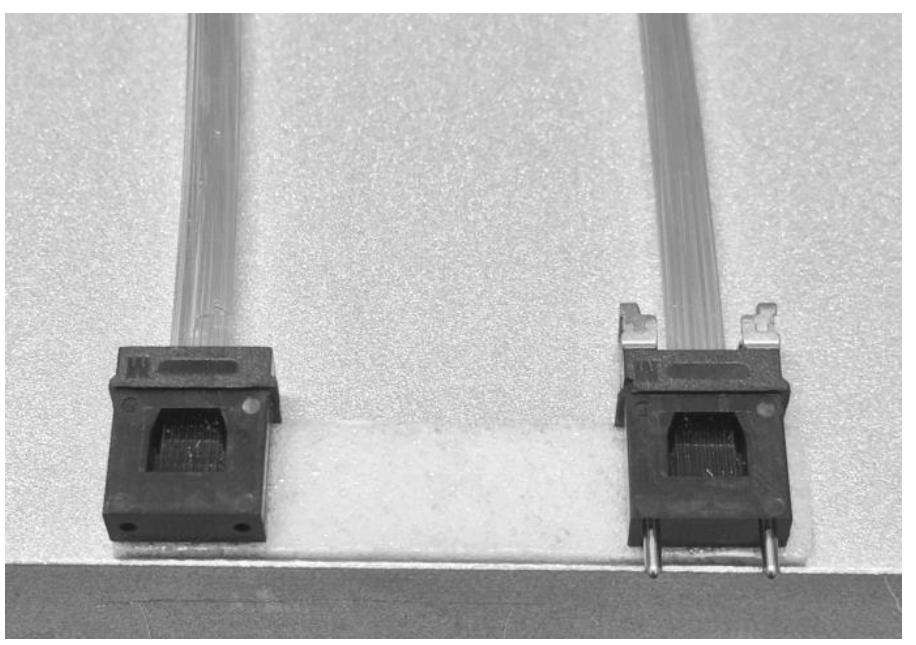

Figure 2- Photograph of the USCONEC 32MTP ferrules, female and male.

\section{FRD - METHODOLOGY}

The major problem in the cone FRD methodology process is related to possible angular errors which may have different causes. In fact, angular deviations between the face of the fiber and its optical axis come from manufacturing or polishing errors as we will see throughout this paper. However, pointing errors in the FRD measurement experiment are quite critical in that they may lead to erroneous conclusions about the conditions of the fibers under test. In fact, this problem is often observed in tests that, like ours, use cones of light with controlled focal ratio ${ }^{(05,06)}$. FRD analyzes can be made through the Absolute Transmission curves analysis ${ }^{(07,08)}$. These are obtained through the integration of encircled energy as it asymptotically approaches the total, but it is difficult to do very accurately. For this reason, this technique is not sensitive enough to detect and measure angular deviations. In this work, we have used a very direct technique to evaluate the FRD, which is much more reliable than the conventional technique using the integrated enclosed energy, and, as we shall see, produce a diagnostic of much more subtle effects from the data obtained. In our current experiments, the FRD is determined straightforwardly from the images, by investigating the form of the edge of the illuminated disk. In the absence of FRD that edge is infinitely sharp, and indeed the edge is very sharp in the reference images. In this way, we basically analyze the radial profile of the image projected by the optical fiber and collected by a CCD. Since the profile is 
not exactly the same in an angular distribution around the center of the image, we take the mean profile obtained in 18 sectors of 20 degrees each. The slope analysis of the curve obtained by this process is quite sensitive to angular deviations of a cone of light at the entrance of the fiber under test. However, this procedure requires a meticulous computational task to define the center coordinates of the image with adequate accuracy.

\section{1 - Input-Output Geometry}

In order to establish our process of analysis we start from the principle of two previously observed conditions in optical fibers. If a light ray is applied to the entrance of an optical fiber at some azimuthal angle, and with some polar angle $\alpha$ with respect to the axis of the fiber, it will ideally emerge from the fiber at the same azimuthal and polar angles. In practice, however, the azimuthal angle on emergence varies so rapidly with, the length and diameter of the fiber, that the emerging ray spreads to fill an annulus of a cone of twice angle $\alpha$, as shown in Fig. 3. Therefore, when a solid cone of light is applied to the entrance of an optical fiber such that the axis of the cone coincides with the optical axis of the fiber the projection of light at the outlet will also be a solid cone of light as shown in Fig. 4. The output cone will also have its axis coincident with the optical axis of the fiber, since we are not initially considering prismatic effects. It is also true that for an ideal fiber in the absence of FRD the output light cone is a reproduction of the light cone of the input.

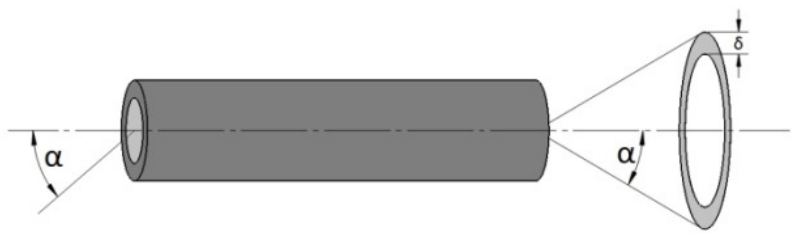

Figure 3- Condition observed in a fiber fed with a beam having no divergence, i.e. the input beam has $\mathrm{NA}=0$. Incorrect aiming with respect to the optical axis of the fiber results in a ring of light at the input angle.

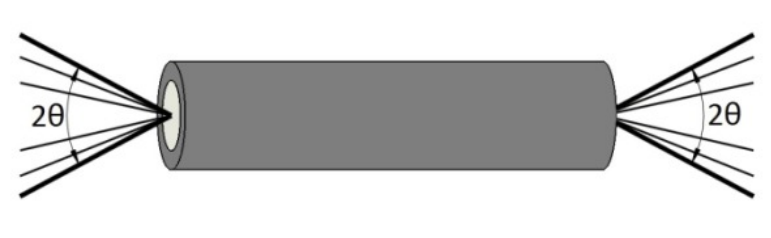

Figure 4- Condition observed in a fiber fed with a cone of light. In an ideal optical fiber, without any kind of FRD, the output reproduces exactly the same cone of light applied at the input.

Both situations exhibit basic operational characteristics of optical fibers without taking into account prismatic effects in both the input and the output of the fibers. Prismatic effects occur as a function of angular deviations between the optical axis of the fiber and the normal vector of its polished surface. A light cone experiment for FRD evaluation should take into account both the prismatic effects of the optical fiber and the aiming error of light cone. In the latter case, it is characterized by the mismatch between the axis of the cone and the optical axis of the fiber under test. This is a mixture of the two basic conditions described above. The Fig. 5 shows this combination.

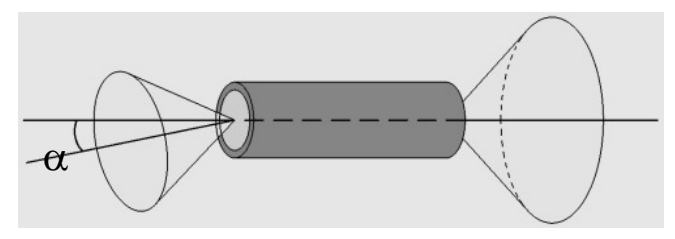

Figure 5- A combination of the conditions shown in Figs. 2 and 3 is obtained by feeding the input of the optical fiber with a solid cone of light such that the axis of the cone does not coincide with the optical axis of the fiber. The consequence is the widening of the cone of light projected by the fiber exit.

Obviously, a suitable procedure is required to align the polished surface of the optical fiber with the light cone of the experiment, as we are going to discuss in detail in the experimental procedure. However, it is also necessary to develop a mathematical process that allows us to evaluate how much the angular deviation between the optical axis of the fiber and the normal vector of its polished surface changes the FRD. 


\section{2 - The FRD basic Analysis}

Our model takes in account FRD Gaussian scattering, so that the effect of FRD on the angular distribution of light emerging from the fiber is given by convolving the illuminated disk on the detector (in units of NA), with a 2-d Gaussian with width parameter $\sigma$ measured in NA units. This will clearly just soften the edge of the disk, and if the disk is very large or if $\sigma$ is very small, the form of the edge is just the complement of the normal distribution function with parameter $\sigma$ and argument the distance (in NA) from the edge.

Let $\boldsymbol{R}$ be the radius of the disk unaffected by FRD. This is approximately $\boldsymbol{r h}$, the radius at half the mean interior intensity, but is not exactly because of some geometric effects; we can determine the difference. $\boldsymbol{r}$ is the radius at which we measure the intensity and $\sigma$ the Gaussian width parameter in pixels. In dealing with pixelated images of this disk, we will for convenience discuss the quantities as measured in units of pixels. This is related to $\sigma$ simply by the scale factor relating pixels to NA. Let $\boldsymbol{R}-\boldsymbol{r}$ be the distance from the point of interest to the periphery of the disk. The geometry is sketched in Fig. 6.

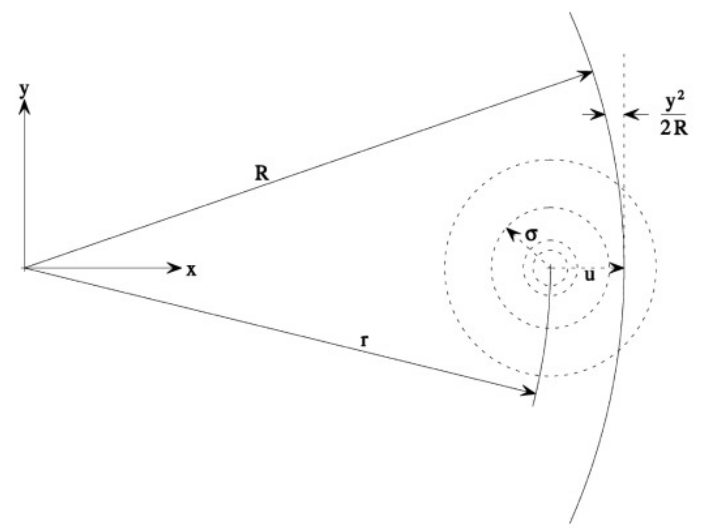

Figure 6- Sketch of the geometry for the FRD effect on the angular distribution of light from a fiber. In the absence of FRD, the disk of radius $\mathrm{R}$ in NA is uniformly illuminated (provided the input is uniform). FRD smears the intensity, in our model by a Gaussian kernel of width parameter $\sigma$. Clearly if the radius R is very large compared to $\sigma$, the distribution with no FRD $(\sigma=0)$ is just a step function in $\mathrm{u}$ which goes from 0 to 1 at $\mathrm{u}=0$ for increasing $\mathrm{u}$ (coming in). For nonzero $\sigma$, the function is simply the normal distribution function in $u$ with parameter $\sigma$. For finite R, this function is modified, but in a very simple manner, as we demonstrate in the text.

If $\boldsymbol{\sigma} / \mathbf{R}$ is small, which we will assume in all the following, it is clear that, for $|\boldsymbol{y}|$ not much larger than $\boldsymbol{\sigma}$, the distance from the extreme point on the circle to a point at height y is $y^{2} /(2 R)$, as shown in the drawing. Since the kernel is Gaussian, almost all the weight is within a few $\boldsymbol{\sigma}$ of the center in both directions. Let $\boldsymbol{F}(\boldsymbol{u})$ be the intensity at $\boldsymbol{u}=\boldsymbol{R}-\boldsymbol{r}$, assuming the intensity in the interior is unity and that $\sigma / \boldsymbol{R}$ is small. Then

$$
\begin{aligned}
F(u) & \approx \frac{1}{2 \pi \sigma^{2}} \int_{-\infty}^{\infty} e^{-y^{2} / 2 \sigma^{2}} \int_{-\infty}^{u-y^{2} / 2 R} e^{-x^{2} / 2 \sigma^{2}} d x d y \\
& \approx \frac{1}{2 \pi \sigma^{2}} \int_{-\infty}^{\infty} e^{-y^{2} / 2 \sigma^{2}} \int_{-\infty}^{u} e^{-x^{2} / 2 \sigma^{2}} d x d y-\frac{1}{2 \pi \sigma^{2}} \int_{-\infty}^{\infty} e^{-y^{2} / 2 \sigma^{2}} \int_{0}^{y^{2} / 2 R} e^{-(u-z)^{2} / 2 \sigma^{2}} d z d y
\end{aligned}
$$

Since we assume that $\sigma$ is much smaller than $R$, it follows that $\sigma^{2} / \boldsymbol{R}$ is much smaller than $\sigma$, so the integrand in the z part of the second integral is essentially constant over the range of integration and is just $e^{-\left(u^{2} / 2 \sigma^{2}\right)}$. Thus, the double integral just integrates $y^{2} / 2 R$ with the Gaussian kernel, yielding $\sigma / 2 R$. There are the normalizing factors of $2 \pi$. The y integral in the first term is unity and the $\mathrm{x}$ integral is the normal distribution function, $\boldsymbol{n d f}(\boldsymbol{u} / \boldsymbol{\sigma})$. The final result is

$$
F(u) \approx n d f\left(\left[u-\sigma^{2} / 2 R\right] / \sigma\right)
$$


The curvature of the edge of the illuminated disk means that the y-integral is summing the normal distribution function at arguments from $\mathrm{u}$ to $\boldsymbol{u}-\boldsymbol{y}^{2} / 2 \boldsymbol{R}$, i.e. the function shifted by these amounts. As long as the function is changing over a scale large compared to the shifts, i.e. $\boldsymbol{y}^{2} / 2 \boldsymbol{R}<<\sigma$, this superposition just results in a function with some mean shift, and the mean shift is simply the shift evaluated at the second moment in $\boldsymbol{y}, \boldsymbol{\sigma} \boldsymbol{\boldsymbol { 2 }}$. Thus, the form of the intensity is still the normal distribution function, but shifted inward by the small correction $\sigma^{2} / 2 R$, or a fractional shift in radius of $\sigma^{2} / 2 R^{2}$. In NA units, for $\boldsymbol{R} \sim 0.2$ and $\boldsymbol{\sigma} \sim \mathbf{0 . 0 2}$, this is half a percent in radius and the approximations we have used are fine. The next order term is of order $\boldsymbol{\sigma} / \boldsymbol{R}$ times this, or about a ten percent correction to this half percent effect. Thus, the intensity expressed as a function of $r$ is

$$
\begin{aligned}
& I(r)=F(R-u)=n d f\left(\frac{R-r}{\sigma}-\sigma / 2 R\right), \\
& \text { or, since } \mathrm{n}(0)=1 / 2, \text { we can write, simply, } \\
& I(r)=n d f\left(\frac{r h-r}{\sigma}\right),
\end{aligned}
$$

where $\boldsymbol{r}_{\boldsymbol{h}}$, again, is the radius at which the intensity drops to half its mean value. Clearly, we have $\quad \boldsymbol{r}_{\boldsymbol{h}}=\boldsymbol{R}-\boldsymbol{\sigma}^{\mathbf{2}} / \mathbf{2} \boldsymbol{R}$. In practice, we could either do a nonlinear least-squares fit to the measured profile or pick a couple of values of intensity, one near zero and the other near 1, find the radii at which those values occur, and choose $\sigma$ so that $\boldsymbol{n d f}\left(\left[\boldsymbol{r}_{\boldsymbol{h}}-\boldsymbol{r}\right] / \boldsymbol{\sigma}\right)$ goes through those values. In fact, we can use this simpler technique, and have chosen symmetric values of 0.85 and 0.15 of the mean intensity. For our model, the difference in radii at these values is $\mathbf{2 . 0 7 \sigma}$, and we call the $\sigma$ so derived or $\sigma_{\text {тот }}$. The basic profile $\boldsymbol{F}(\boldsymbol{\theta})$ with no angular misalignment is the complement $\boldsymbol{n} \boldsymbol{d f \boldsymbol { c }}\left(\left[\boldsymbol{r}-\boldsymbol{r}_{\boldsymbol{h}}\right] / \boldsymbol{\sigma}\right)$ of the normal distribution $\boldsymbol{n d f}\left(\left[\boldsymbol{r}_{\boldsymbol{h}}-\boldsymbol{r}\right] / \boldsymbol{\sigma}\right)$, where $\boldsymbol{n d f}=\boldsymbol{0 . 5 *} \operatorname{erf}\left(\left[\boldsymbol{r}_{\boldsymbol{h}}-\boldsymbol{r}\right] / \boldsymbol{\sigma}\right)+\boldsymbol{0 . 5}$. Then we can write;

$F O(r)=n d f c((r-r h) / \sigma)$

\section{3 - Adding Angular Error at the Input}

A regular situation defined by an angular error input, whether because of the polishing at an angle or because of the misalignment of the experiment, is exactly a mixture of the two specific situations described in section 1.1, Fig.3, with the addition of FRD. It is therefore reasonable to assume that in the presence of FRD, if the input light cone is not on the axis of the fiber, the result at the output is the input cone convolved with the ring described in the Fig. 1, and convolved with the FRD. This convolution is just a sum defined by:

$F A=\sum_{i} w t(i) * n d f c(r, r h, \sigma T O T, A, i)$

Where $\boldsymbol{w t}(\boldsymbol{i})$ are composed by the discrete terms obtained from the 1-d kernel for a ring with angle $\alpha$, very well represented by the expression,

$K(x)=\frac{C}{\sqrt{1-\frac{x^{2}}{\alpha^{2}}}}$

So, we start with the specification of (04), defining how many terms are needed to get satisfactory accuracy. The parameter $\boldsymbol{A}$ is defined by:

$A=\alpha / \sigma_{F R D}$ 
if this is small, the convolution kernel is very narrow and a sum of a few terms will do, and if large it is wide and we will require many terms. For our purposes, a range of $\boldsymbol{A}$ from zero to seven terms will do. So, all one needs are the seven coefficients to represent the convolution integral. Since it is symmetric, there are only four different values. Then, the array $\boldsymbol{w t}(\boldsymbol{i})$ can be produced by integrating the kernel exactly over cells and then normalizing so the sum is unity. The singularities are placed at the centers of the end cells, so the integration is only over the interior halves of those cells with width 6, Fig. 7. In a discrete convolution, as suggested, the ndf c function must be discretized in (i) by the same length of $\boldsymbol{w t}(\boldsymbol{i})$. The term to be discretized is obviously the misalignment parameter A.

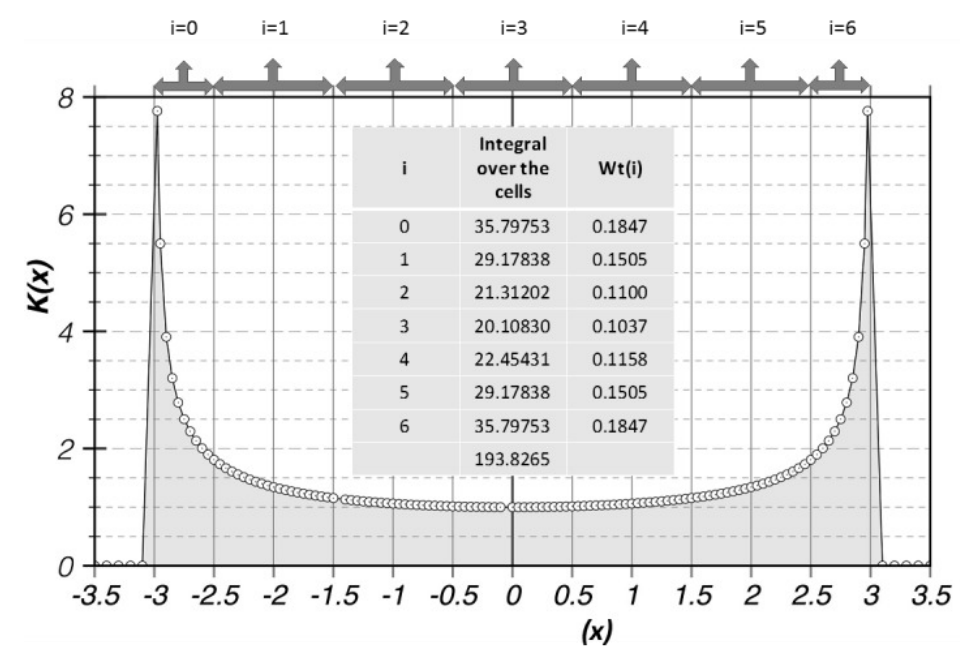

Figure 7 - Process to integrate the kernel exactly over the cells. The last number in the center of the table come from the sum of the central column terms and is used to normalize each term of this column. The results are the elements of wt(i).

With these observations in mind the expression (04) is now specified by,

Fcon $=\sum_{0}^{6} w t[i] * n d f c\left[\left(\frac{r-r h}{\sigma_{T O T}}\right)+(i-3) * A / 3\right]$

The effect of this convolution generates a profile characterized by the misalignment defined by $\boldsymbol{A}$. However, the generated curve will have its width changed also as a function of $\boldsymbol{A}$. In other words, as $\boldsymbol{A}$ increases the width defined by wid also Increases. Our goal is to generate curves with the same wid value, so that it is necessary to define a compensation factor wapp that multiplied by $\left(\frac{r-r h}{\sigma_{T O T}}\right)$ holds the wid width of the profile generated by the convolution constant. So, we will have the final expression;

$F A\left(r, r h, \sigma_{T O T}, A\right)=\sum_{0}^{6} w t[i] * n d f c\left[\left(\frac{r-r h}{\sigma_{T O T}}\right) * w a p p+(i-3) * A / 3\right]$

But, how to obtain wapp as function of $\boldsymbol{A}$ ? Making sigma as unity for the function in (9), the parameter A is just the angular misalignment normalized to sigma $=1$, and it means the same thing here as it does for real data. So, if we convolve $\boldsymbol{n d f} \boldsymbol{c}(\boldsymbol{u})$ with the misalignment kernel we will get a new function with a larger wid and wid is 2. 07289.. for the un-convolved function. To understand how the wid changes with $\boldsymbol{A}$ we just need to normalize the family of functions that we will fit with to the same wid. Investigations with essentially exact integrations, can give us results for discrete values of $\boldsymbol{A}$. However, 
wapp values can be obtained by the function;

$w a p p=\sqrt[2]{1+\frac{A^{2}\left(1+A^{2}\right)}{\left(2+1.5 A^{2}\right)}}$

Since in any convolution the RMS widths add in quadrature, and for a Gaussian the $r \boldsymbol{r} \boldsymbol{s}$ width is just $\boldsymbol{\sigma}$ and for the ring kernel is $\alpha / \sqrt{ } 2$, we have,

$$
\sigma_{T O T}^{2}=\sigma_{F R D}^{2}+a^{2} / 2
$$

Where $\sigma_{\text {тот }}$ is the RMS width of the convolved kernel with both FRD and misalignment. We do not, however, really measure the RMS width of the kernel unless the kernel is accurately Gaussian. For a much smaller than $\sigma_{F R D}$, the kernel is still essentially Gaussian, so we expect this to hold however we measure the total width, but not for the dominant misalignment we see. There should, however, still be a quadratic relationship, but what will change is the numerical factor for $\alpha^{2}$. Numerical experiments for $\alpha$ of the order of $\sigma$ or larger and measuring $\sigma_{\text {тот }}$ the way we do, by measuring the width of the $85 \%$ to $15 \%$ transition region in the profiles, yields the result that the simple relation,

$$
a^{2} \approx 1.5\left(\sigma_{T O T}^{2}-\sigma_{F R D}^{2}\right)
$$

reproduces the measured quantities to within a few percent. This result allows us to accurately create simple families of theoretical profiles with fixed total width as measured by our estimate of $\sigma_{T O T}$ with variable input guesses for $\sigma_{F R D}$ to compare in detail with the observed profile. Then, it is possible find the best fit by least-squares over the radial range $\mathrm{r} 1$ to $\mathrm{r} 2$ as one varies A from 0 to some value from the table. Following this procedure, we find the best $\boldsymbol{A}$, which is defined by $\alpha / \sigma$, so we have

$\alpha=A * \sigma_{F R D} \Rightarrow \alpha^{2}=A^{2} * \sigma_{F R D}^{2}$

Finally, from (10), (12) and (13) we can write:

$\sigma_{T O T}=\sigma_{F R D} * w a p p$

\section{EXPERIMENTAL PROCEDURE}

The diagram of the experiment is shown in Fig. 8. The primary light source of this experiment is a large LED lamp $\mathbf{S}$, which light is diffused by $\mathbf{D}$, and condensed to illuminate a pinhole device $\mathbf{P}$. A filter $\mathbf{F}$, centered at $550 \mathrm{~nm}$ defines the wavelength in which we are working on. The pinhole is the secondary light source and it projects light to be collimated using a lens device. A set of lenses $\mathbf{O B J}$, converges the collimated beam generating a cone of light with NA well defined by the diameter of the diaphragm DIA, to be inserted into the input of the test fiber. The CCD $\mathbf{1}$ take image from the test fiber input surface, by a beam splitter BS. This one allows to observe accurately the position of the test fiber using a TV monitor. A displacement device POS, defines the alignment of the fiber in test and the CCD 2, when it is necessary take reference images from the source. 


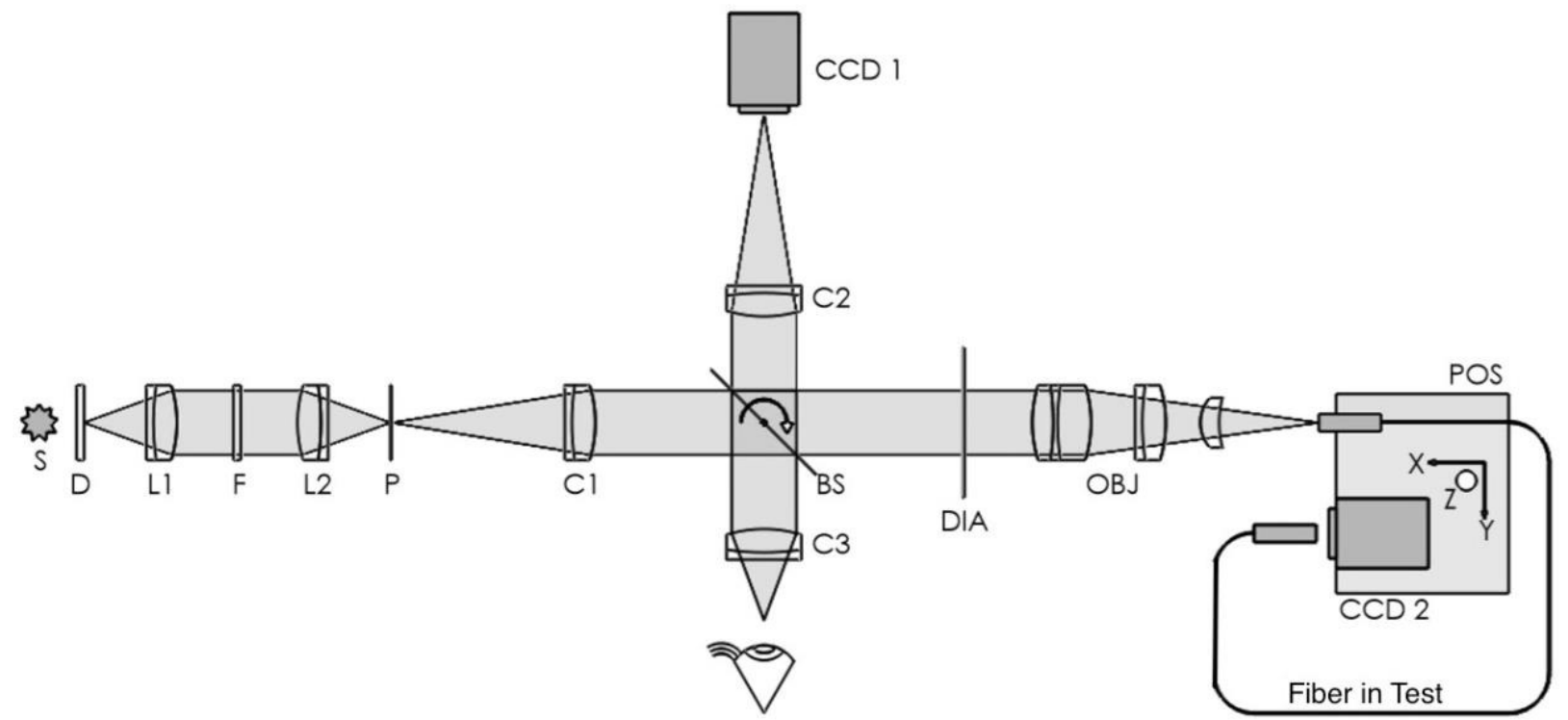

Figure 8- Diagram of the apparatus used to measure FRD in optical fibers. S, extensive LED lamp; F, filter; P, Pinhole; C1, collimator lens; BS, beam splitter; CCD 1, alignment CCD; DIA, adjustable iris diaphragm; OBJ, camera lens; CCD 2, CCD detector; POS, xyz translation stage; $\mathrm{C} 2$, focusing lens for the test fiber; $\mathrm{C} 3$, focusing lens for the diaphragm.

The focal ratio of this experiment was defined in F / 2.8 by standardizing all tests with a numerical aperture of 0.1785 at the entrance of the fibers. For a high performance polished surface optical fiber, the alignment process can be done by reflecting the light cone through the surface of the polished fiber. In this work, we present a very simple procedure that involves only a good lens device $\mathbf{C 3}$, with adequate magnification. The beam splitter BS can rotate 90 degrees and can change the refection from the fiber to $\mathrm{C} 2$ or $\mathrm{C} 3 . \mathrm{C} 2$ is calculated to produce an image focused in the fiber input surface. $\mathrm{C} 3$, on the other hand, is calculated to produce an image focused at the DIA. When the beam Splitter is rotated to reflect the diaphragm image through $\mathrm{C} 3$, it is possible to observe the reflection of light incident on the core of the fiber under test. This surface behaves like a flat mirror and reflects about $2 \%$ of the incident light. If the normal vector of the polished surface is perfectly aligned with the axis of the cone of light, then the reflected beam returns exactly on the incident beam. By this way, it is possible to observe the fully illuminated diaphragm homogeneously, Fig 9(a). However, if the normal vector of the polished surface has an angular deviation from the axis of the cone of light, then the reflected beam does not return exactly on the incident beam. In this case, the observed image does not completely fill the diaphragm field, Fig. 9(b, $\mathrm{c}, \mathrm{d}, \mathrm{e})$.

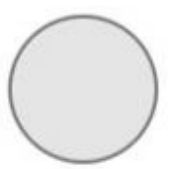

(a)

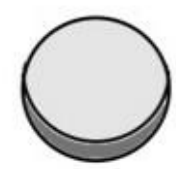

(b)

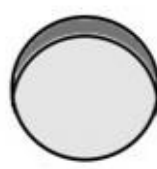

(c)

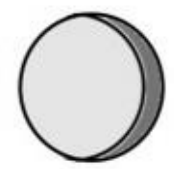

(d)

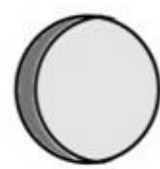

(e)
Figure 9- Diaphragm retro-illuminated by reflection of incident light on the surface of the fiber on test. In (a) the diaphragm space is fully illuminated because the reflected beam returns exactly on the optical axis of the light cone. In (b), (c), (d) and (e) the diaphragm space is not fully filled because there is a deviation between the axes of the incident light and the light reflected by the fiber. The angular deviation combinations shown are on the vertical and horizontal axis, but may obviously be presented as combinations of these examples. 


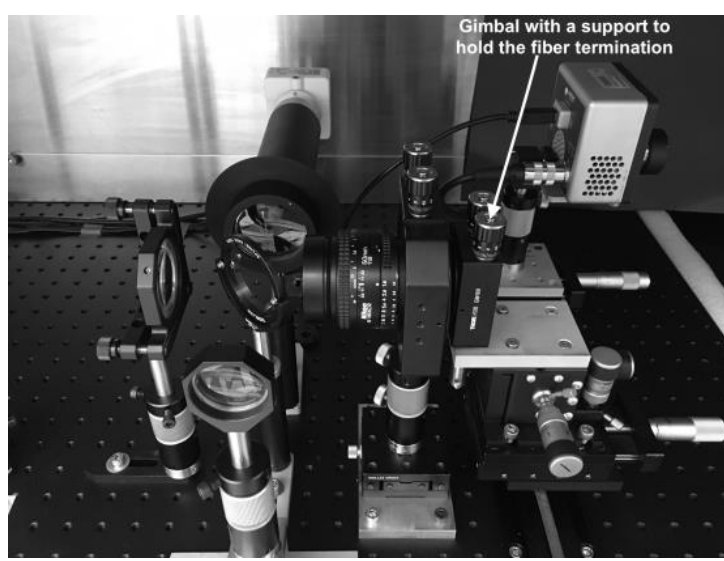

Figure 10- Gimbal where the termination of the optical fiber under test is engaged.

Checking by eye using a lens with correct magnification is easy and quite simple to do. The ferrule containing the termination of the optical fiber under test is fixed by means of a quick coupling bracket inserted inside a Thorlabs Gimbal device, Fig.10. This bracket is a support that can be rotated so that the image of Fig. 6 can be viewed with horizontal or vertical symmetry. The correct adjustment of the Gimbal allows to correct the angular deviation until it is possible to observe the image of the area of the diaphragm completely illuminated. Once made this adjustment, we can be absolutely sure that the axis of the light cone is aligned with the normal vector of the optical fiber polished surface. Obviously if the polishing was performed without angular error, then the axis of the light cone will also be aligned with the of the optical fiber axis. This is the starting point for a detailed analysis of the effects caused by angular errors of polishing since with this reference factor, we can isolate the pointing error of the polishing angular error. In other words, with respect to the optical axis of the fiber, both angular deviations are the same thing and therefore without previous alignment we would be unable to treat and quantify the effects within a FRD analysis perspective. This procedure proved to be extremely effective and accurate, while not requiring too much space or long optical paths to be installed.

The proper combination between the Pinhole $\mathrm{P}$ diameter, the $\mathrm{C} 1$ and OBJ lenses define the spotlight size projected on the test fiber. A spotlight with a diameter much smaller than the diameter of the core of the fiber causes the light output of the fiber to exhibit a type of Annular Degeneration shown in Fig. 11(a). On the other hand, a spotlight on the fiber with a diameter larger than the diameter of its core makes the light output of the fiber producing Cladding Modes, Fig. 11(b). The best option is to approximate the spotlight diameter between $95 \%$ and $100 \%$ of the core diameter of the fiber under test. This can be significantly complicated mainly because of the industrial limitations of the commercial Pinholes diameter. However, an effort to bring the spotlight diameter as close as possible to the core diameter, ensures greater homogeneity in the fiber optic light output as shown in Fig.11(c).

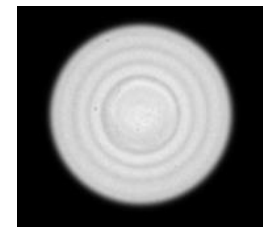

(a)

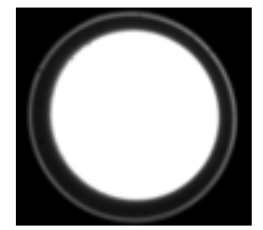

(b)

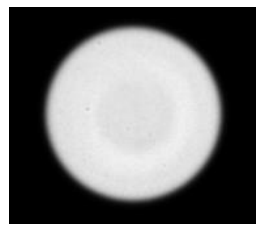

(c)
Figure 11- Depending on the diameter of the spotlight projected onto the input surface of the fiber under test, the disk of light projected by the fiber outlet may exhibit anomalies in its radial profile. The best condition, (c), is obtained when the diameter of the spotlight is almost exactly the same as the diameter of the fiber core.

Eventually the image obtained at the output of the optical fiber may not be perfectly homogeneous. This can happen because of anomalies in the radial profile of the light disk designed by the light cone that feeds the optical fiber under test when it is very short, less than 2 meters. Most often the result is a bump in the central region of the disc, which must be eliminated from the radial analysis processes to avoid errors in the calculation of SigTOT and SigFRD values.

\section{1 - CCD 2- lens}

The CCD 2 used in this work was the Andor Solis DL-604 M-OEM. This CCD is 1000 x 1000 pixels with 8-micron size pixel, so it is $8 \mathrm{~mm}$ on the side. We have calculated a lens system such that the projected image size in the CCD is of the order of $5 \mathrm{~mm}$ in diameter at a focal ratio $\mathrm{F} / 2.8$. The system is composed by symmetrical doublets, Fig. 12a. The performance of this doublet is extremely good; The deviations of these rays are less than 1.2 milliradians from the design 
goal. In terms of the location of the CCD image, this is an error of about 17 microns in $5 \mathrm{~mm}$ - corresponding to the same 1.2 milliradians in angular offset. The lenses are housed in a camera where one side is coupled to the $\mathrm{C}$-mounting and the other side is constituted by a holder which holds the ferrule with the polished fiber termination or USCONEC ferrule connector Figs. 12b and 12c. This support can be changed depending on the type of ferrule in the sample being tested. In all cases however, such a holder holds the end of the optical fiber exactly in the object position defined by the optical design of the system. Once the ferrule is inserted into the holder, almost all ambient light is locked onto the CCD. This allows for a fairly efficient Dark even with some diffused light in the experimental environment.
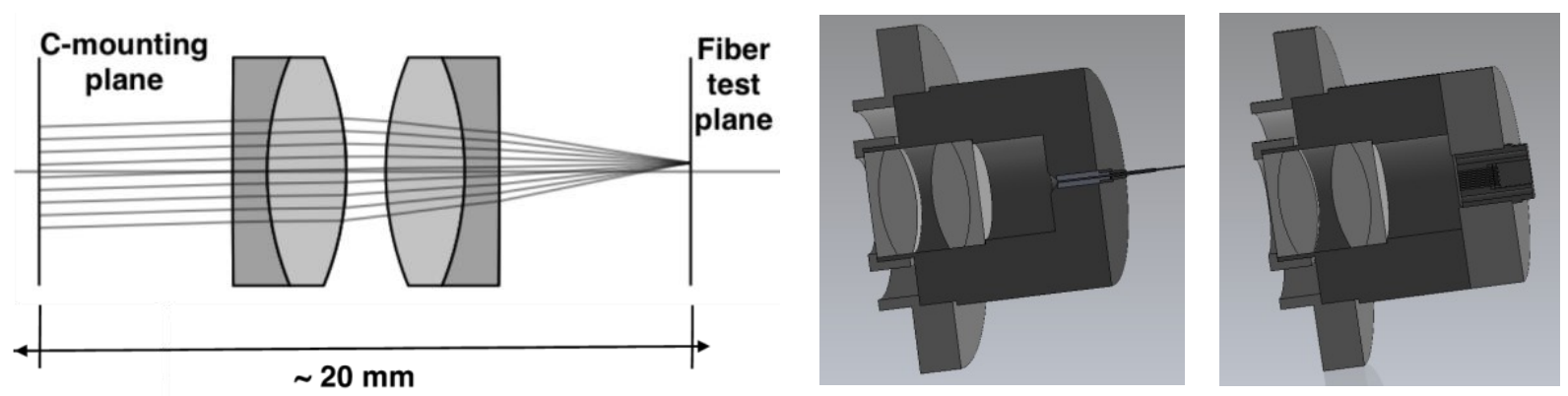

Figure 12 - (a) - Symmetrical doublets used at the entrance of CCD2. The total length between the position of the fiber under test and the plane of the C-mounting is about $20 \mathrm{~mm}$. (b) - Camera adapted for insertion of zirconia ferrule. (c) Camera adapted for insertion of USCONEC ferrule.

\section{2 - Data Reduction}

We have developed codes in different programming languages which show similar results, using images signed in 16 bits with. fits format. Based on the considerations in the previous sections, special care is taken in the logical analysis. The code logic should basically follow the sequence:

- Subtracting Dark from images

- Find a center with the best possible precision

- Obtain radial profiles in at least 18 angular sectors of the image

- Obtain the average of these angular profiles

- Delete the central region eventually discontinued

- Calculate the Sigma TOT

- Calculate possible values of Sigma FRD with respective misalignments

- Plot the curves corresponding to previously calculated Sigma FRD values.

- Choose the curve that best fit with the original profile obtained with Sigma TOT

- Find the correct value of FRD and corresponding misalignment

The final result allows us to accurately create simple families of theoretical profiles with fixed total width as measured by our estimate of Sigma TOT with variable input guesses for Sigma FRD to compare in detail with the observed profiles.

\section{RESULTS AND DISCUSSION}

When we can be sure that the polishing was performed in such a way that the normal vector of the polished surface is parallel to the optical axis of the fiber, all we have to do is align the optical axis of the fiber with the axis of cone of light. To do this, we perform the procedure described in the previous section thus ensuring that the light reflected by the 
optical fiber fills the entire diaphragm. There are instruments that can evaluate and measure any angular deviation after the polishing of the ferrule that contains the fiber. There are also laboratory procedures that allow to measure indirectly whether there is any angular error of polishing. However, for most professional polishing jigs, the angular deviation is very close to zero if not completely zero to what we can measure. In general, the angular error is caused either by manufacturing defect of the ferrule or by improper calculation between the diameter of the fiber and the diameter of the ferrule channel. The zirconia ferrule that should be used in the FOCCoS / PFS project was very well designed. The difference between the diameter of the optical fiber and the diameter of the ferrule hole is about 3\%, which defines an angular deviation of less than 0.0009 rads. This deviation is not significant for FRD analysis and indeed it is within the accuracy of evaluation by eye we propose here. The Fig. 13 shows the curve obtained from a fiber sample terminated with zirconia ferrule in both extremities. The theoretical curve represents a situation with zero angular deviation and was obtained based on the equation (04) of the theoretical analysis described in section 2.2. Since the theoretical and experimental curves are practically coincident in the considered interval we can affirm that the fiber in question does not present measurable angular deviation from assembly or polishing errors. In fact, the curve obtained in Fig.13, is very well behaved, a characteristic situation of a correct alignment between the polished face of the fiber, the optical axis of the fiber and the axis of the cone of light. In such a situation, the value of SigmaTOT is equivalent to SigmaFRD so that the evaluation of samples like these can be done simply and directly. The Fig.14, shows the curves obtained from a sample with SigmaTOT significantly higher than the first sample. Even when there is an increment of FRD for any reason that is not mimicked by angular deviation, the theoretical Gaussian profile curve almost perfectly matches the experimental curve in the given interval. We have evaluated hundreds of samples with these. The histogram of Fig. 15 shows the statistical distribution of the Sigma values, obtained

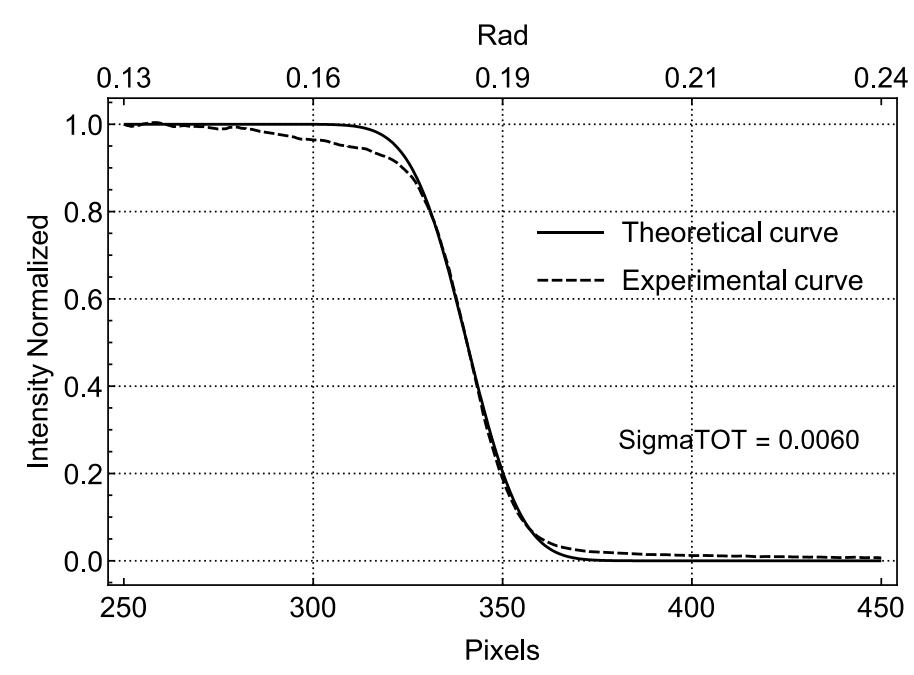

Figure 13- The figure shows two curves referring to a Polymicro FBP127168190 fiber sample of about 17 meters in length with both ends inserted in zirconia ferrules. The dashed curve is the experimental curve of the radial profile of the fiber, normalized with the mean of pixels' values sum between 295 and 300. The continuous curve is the theoretical curve generated with the same characteristics $\boldsymbol{w d}, \boldsymbol{r h}$ and SigmaTOT. The similarity of the two curves is quite significant. The representation is made between the pixels 250 and 450 , where we find the values of 0.15 and 0.85 of the $\mathrm{Y}$ axis for the calculation of SigmaTOT. matching the normal vector of the polished surface of the fiber with the axis of the cone of light through the observation of the pupil by the lens $\mathrm{C} 3$ of the experiment. from images obtained from 240 fiber samples prepared and polished under the same conditions. This distribution tells us that more than half of the fibers exhibit Sigma values between 0.007 and 0.010 and that fewer than 50 fibers exhibit values greater than 0.010 . These values can be used to characterize the FRD of the samples and subsequent choice of the fibers that fit the desired range as a function of the optical design of the system. It is interesting to note that these values are specifically determined by the FRD conditions with no measurable contribution of angular deviation and therefore represent SigmaFRD. To be sure of this condition all experimental curves are juxtaposed with the respective theoretical curves and all showed a behavior similar to the examples plotted in Figs. 13 and 14. On the other hand, the situation is quite different when there is a significant angular deviation. We can see this clearly in images obtained from optical fibers inserted into USCONEC ferrules. Several samples of cables with fibers inserted into these connecting cells were prepared and studied. The preparation was done with all possible care to avoid stress and the polishing was carried out using high precision industrial jigs. However, a systematic angular deviation was observed in all samples. This basically means the non-overlap between the theoretical curve and the experimental curve. In the same way as before, the initial alignment is done by 
However, since the optical axis of the fiber does not coincide with the normal vector of the polished surface, this simple procedure does not eliminate the error due to the angular deviation. But from this procedure, it is possible to change the angle of the optical axis in a controlled way by adjusting the Gimbal. A simple sequence of adjustments enables the correct alignment of the optical axis of the fiber to the axis of the cone of light to be found from the initial alignment of the normal vector of the polished surface to the axis of the cone of light. In Fig. 16 we show the image and radial profile of a fiber with both ends inserted in USCONEC ferrules. The analysis of the radial profile, experimental curve, shows the characteristic behavior of angular misalignment between the optical axis of the fiber and the axis of the cone of light that

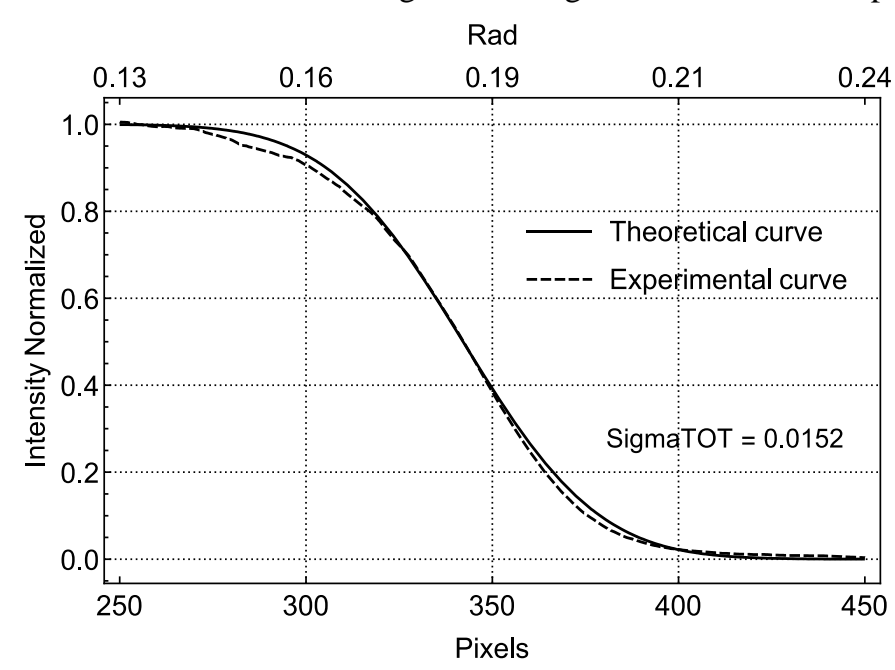

Figure 14- This is a sample that presents SigmaTOT approximately twice as large as the first sample. However, the experimental curve remains well-behaved showing a good alignment between the optical fiber axis, the normal vector of the polished surface and the axis of the light cone.

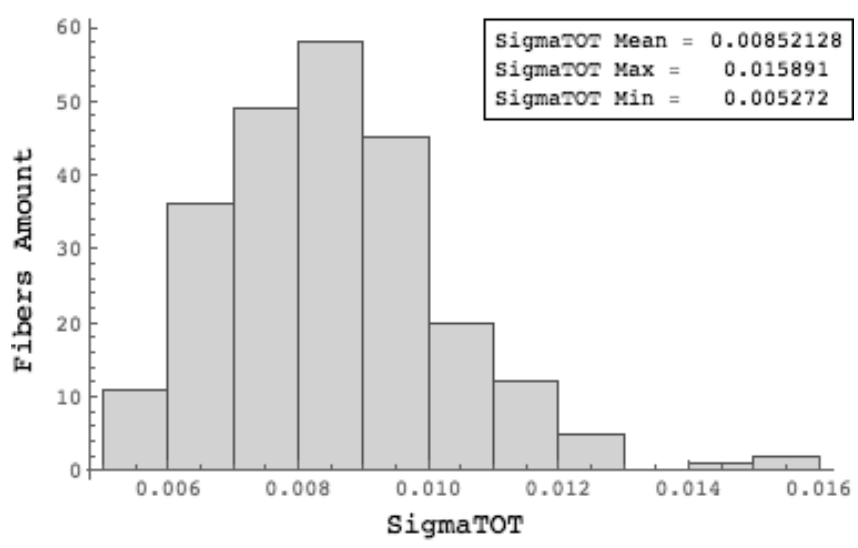

Figure 15- Histogram of statistical distribution obtained from 240 fibers samples equally prepared. feeds the fiber. A simple procedure of trial and error in Gimbal manipulation, observing the image from the fiber allows to obtain the correct adjustment direction to correctly align the optical axis of the fiber. Fig. 17 shows the result obtained after optimizing the alignment by this proceeding.

Although the readjustment optimization process is efficient and simple as can be attested by these results, it is not practical when it is necessary to characterize tens or hundreds of optical fibers in a short period of time. Mainly because finding the best possible angular alignment between the fiber axis and the cone axis can take considerable time. The application of the mathematical method of image analysis from the misaligned fiber developed in section 3.2 may be more practical under these conditions. Fig. 18 shows the comparison between the experimental curve of the radial profile obtained from the image of the fiber with angular deviation and the calculated theoretical curve that best tape the experimental curve. Recalling that this theoretical curve is calculated based on the SigmaTOT value obtained from the experimental curve. The theoretical curve of Fig. 18 was the curve constructed based on equation (07), section 3.2. Basically, our code constructs several theoretical curves and all of them have the same value of SigmaTOT, but with different combinations of SigmaFRD and Misalignment defined by discretized values of A. Among all of them, the curve that best dresses the experimental curve is the curve which contains the closest value to reality for SigmaFRD. A simple process of point-to-point comparison between the experimental curve and the curves generated, by means of standard deviation, gives us the correct theoretical curve. In this specific case, A $=3.5$ for the theoretical curve obtained. When we compare the value of SigmaTOT obtained from the same fiber shown in Fig. 17, for a correctly readjusted alignment, with the SigmaFRD value obtained through the mathematical method we find a difference of 0.0018 . This is within our current accuracy estimated at 0.002 after measuring a few hundred samples. A better accuracy would require a much better estimate of the mean intensity just before the failure begins, and a much better subtraction background. 

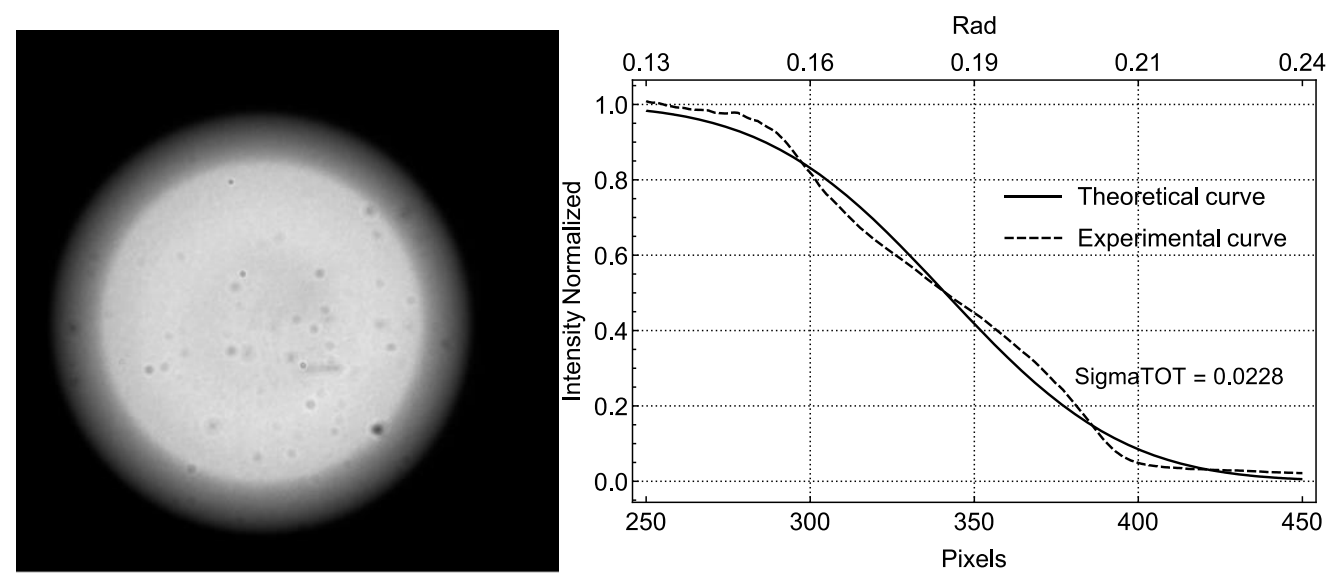

Figure 16- The simple visual analysis of the image on the left shows a luminous halo characteristic of angular deviation at the entrance of the fiber. The curve was obtained from the experimental data obtained from the radial profile of the image. The continuous curve shows what the theoretical profile would be for the same SigmaTOT value.
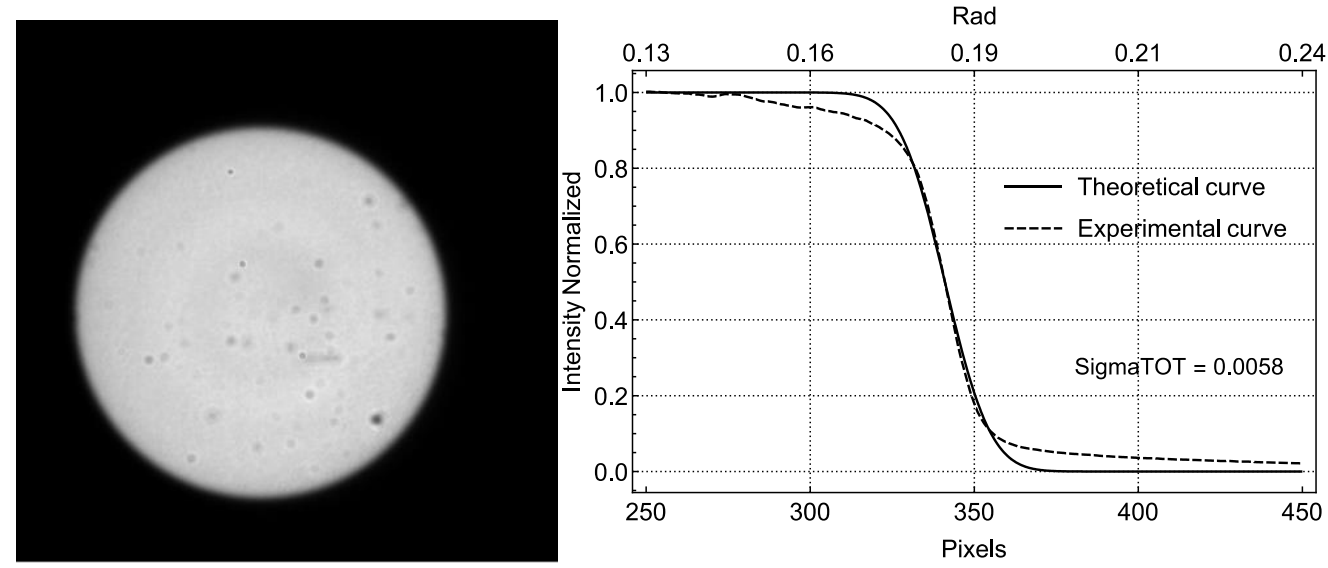

Figure 17- The direction of angular correction in Gimbal can be easily found by visual analysis of the fiber image. However, the correct optimization requires several imaging for various correction increments with subsequent radial profile analysis. The figure shows the image and the angular profile after a complete correction is obtained by this process.

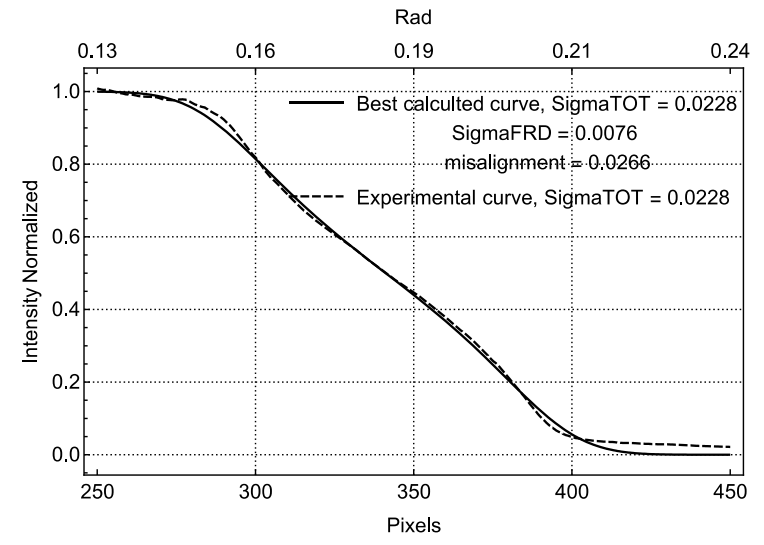

Figure 18- The figure shows the plot of the experimental curve of the radial profile obtained from the fiber image and the plot of the calculated theoretical curve that is closest to the experimental curve. The experimental curve gives us only the value of SigmaTOT, but the curve calculated on the basis of the SigmaTOT value of the experimental curve gives us the values of SigmaFRD and misalignment. 


\section{CONCLUSION}

We have designed a methodology of work to evaluate FRD of optical fibers inserted in ferrules with possible angular errors of assembly or polishing. This methodology consists of a technical procedure of alignment, and a mathematical analysis process which could be used together or separately depending on the demand for samples to be measured. The technical procedure consists of juxtaposing the normal vector of the polished surface of the fiber with the axis of the light cone of the experiment. This is done through light retro-reflection analysis of the fiber under test. In a situation where the fiber has been properly polished exhibiting juxtaposition between its optical axis and the normal vector of its surface, the alignment itself is simple and straightforward showing great accuracy. On the other hand, when there is no coincidence between the normal of the polished surface and the optical axis of the fiber under test a readjustment is necessary. The readjustment consists in finding the correct angle through trial and error in discrete variations of angles at the same time as the decay of the radial profile of the image projected by the fiber is observed. This procedure takes a significant amount of time but can be quite accurate depending only on a Gimbal device correctly adapted to the experiment. The mathematical analysis consists in discretizing the angular error and with this generate several theoretical curves with the same values of SigmaTOT but with values different from SigmaFRD and misalignment. The theoretical curve that best dresses the experimental curve has the SigmaFRD value closer to reality. This procedure can be easily adapted into a code for any type of language and generate results with accuracy less than $0.002 \mathrm{rad}$.

\section{ACKNOWLEDGMENTS}

We gratefully acknowledge support from the Funding Program for World-Leading Innovative R\&D on Science and Technology (FIRST) program "Subaru Measurements of Images and Redshifts (SuMIRe)", CSTP, Japan. The work in Brazil is supported by the FAPESP grant 2012/00800-4 and the Laboratório Nacional de Astrofísica from MCTI.

\section{REFERENCES}

[01] - Oliveira, A.C., et al., "Slit device for FOCCoS - PFS - Subaru" Proc. SPIE 9151, Advances in Optical and Mechanical Technologies for Telescopes and Instrumentation, 91514D, August 07-2014

[02] - Oliveira, A.C., et al., "Fiber optical cable and connector system (FOCCoS) for PFS/ Subaru" SPIE 9151, Advances in Optical and Mechanical Technologies for Telescopes and Instrumentation, 91514G, July 28-2014

[03] - Oliveira, A.C., et al., "Multi-fibers connectors systems for FOCCoS-PFS-Subaru" SPIE 9151, Advances in Optical and Mechanical Technologies for Telescopes and Instrumentation, 915160, July 18-2014

[04] - Haynes, D.M., Haynes, R., Rambold, W., Goodwin, M., Penny, E.J., "Multi-way optical fibre connectors for astronomy" SPIE 7739, Modern Technologies in Space- and Ground-based Telescopes and Instrumentation, 773946, July 2010

[05] - Murphy, J.D., et al., "Focal Ratio Degradation and Transmission in VIRUS-P Optical Fibers" 7018, Advanced Optical and Mechanical Technologies in Telescopes and Instrumentation, 70182T, July 23-2008

[06] - Avila, G., "Tests of optical fibres for astronomical instrumentation at ESO," in [Fiber Optics in Astronomy], Barden, S. C., ed., Astronomical Society of the Pacific Conference Series 3, 63-73 (1988)

[07] - Barden S. C., Ransey L.W., Truax R.J., Evaluation of some fiber optical waveguides for astronomical instrumentation, PASP, v.93, n. 551, p. 154, 1981.

[08] - Wang, R., Yan, Y., Liu, Y., Zhang, H., and Sun, W. (2013). The influence on FRD with different encircled energy. In Optoelectronics and Microelectronics (ICOM), 2013 International Conference on, pages 180-182. 